\title{
Dynamics of Broad-Area Semiconductor Lasers With Short Optical Feedback
}

\author{
Tomokazu Tachikawa, Satoshi Takimoto, Rui Shogenji, and Junji Ohtsubo, Member, IEEE
}

\begin{abstract}
The dynamics of filamentations in broad-area semiconductor lasers with short optical feedback were numerically studied. Regular pulse packages similar to those observed in narrow-stripe edge-emitting semiconductor lasers were found as the feedback reflectivity is increased. However, the regular pulse packages consisted of a periodic envelope of the external cavity frequency and fine pulse trains of periodic filaments, which is different from the regular pulse packages observed in narrow-stripe edge-emitting semiconductor lasers. From the analysis for spatio-temporal near-field patterns, we found periodic undulations of the spatial and temporal filament sizes as the external mirror reflectivity was changed. We also investigated filtered optical feedback, where a part of the emitted beam is fed back into the laser cavity. The time-averaged near-field pattern was found to have a strong dependence on the feedback level in the case of spatial filtered optical feedback.
\end{abstract}

Index Terms-Broad-area semiconductor lasers, chaos, dynamic filamentations, nonlinear optics, short optical feedback.

\section{INTRODUCTION}

H IGH power semiconductor lasers are promising coherent light sources for industrial applications, since they have a higher electrical-to-optical power conversion efficiency than other lasers. One such laser is the broad-area semiconductor laser, which has a similar structure to conventional edge-emitting lasers except that the emitting region has a broad stripe that is up to several hundred microns wide. In such structures, the effects of carrier diffusion and light diffraction in the active region play a critical role in the dynamics. Broad-area semiconductor lasers are unstable light sources due to the spatial dependence of the laser oscillations along the stripe width [1], [2]. While they are capable of emitting at high optical powers, they have rather poor beam quality due to instabilities caused by the microscopic dynamics [3]-[9]. Until recently, most research has focused on how to increase the output power of these lasers and little attention has been given to the dynamics, which are responsible for the deterioration of the beam quality.

Manuscript received May 28, 2009; revised July 23, 2009. Current version published December 09, 2009.

T. Tachikawa was with the Faculty of Engineering, Shizuoka University, Shizuoka, Japan, and is now with Aishin Seiki Co. Ltd., Aichi, 448-8650 Japan (e-mail: f0730311@ipc.shizuoka.ac.jp).

S. Takimoto, R. Shogenji, and J. Ohtsubo are with the Faculty of Engineering, Shizuoka University, Hamamatsu, Shizuoka 432-8561, Japan (e-mail: f0830257@ipc.shizuoka.ac.jp; shogenji@sys.eng.shizuoka.ac.jp; tajohts@ipc.shizuoka.ac.jp).

Color versions of one or more of the figures in this paper are available online at http://ieeexplore.ieee.org.

Digital Object Identifier 10.1109/JQE.2009.2031122
One instability that occurs in the dynamics of broad-area semiconductor lasers is filamentation [3]-[9]. The spatial positions of carrier hole-burning fluctuate with time in the laser cavity and this gives rise to irregular local pulsating oscillations with periods of the order of picoseconds. Since the spatial size of these bright spots is of the order of several microns, broad-area semiconductor lasers exhibit rapid spatio-temporal fluctuations. The presence of filamentations greatly deteriorates the beam quality of the laser, including the time-averaged beam profile and laser coherence. In spite of several investigations, the origin of dynamic filamentations in broad-area semiconductor lasers is not currently fully understood. Also, few studies have been conducted on methods of controlling and stabilizing the filamentations in such lasers. In particular, the relationship between dynamic filamentation and the static beam profile is very important from the viewpoint of applications.

It is widely known that the dynamics of semiconductor lasers are strongly affected by optical feedback, and this is frequently used for beam control. Indeed, the dynamics of broad-area semiconductor lasers subjected to optical feedback have been studied and improvement of the beam quality, including the suppression of dynamic filamentation, has been proposed based on the optical feedback method [10]-[16]. Mandre et al. also investigated a method of control of the spatio-temporal emission by spatially filtered feedback and established the usefulness of this technique [17]. Optical feedback is not the only technique for controlling the spatio-temporal dynamics. The dynamics of broadarea semiconductor lasers are strongly affected by optical injection and it is frequently used to suppress filamentations [18], [19]. In spite of such efforts, quantitative discussions of the dynamics and control of broad-area semiconductor lasers with optical feedback are still required.

In this paper, we consider the dynamics and control of spatio-temporal filaments in broad-area semiconductor lasers subjected to optical feedback in the short external cavity regime. The dynamics of narrow-stripe edge-emitting semiconductor lasers with short external cavities have been studied and some interesting features of the oscillations of regular pulse packages have been demonstrated [20]. The dynamic filaments in a short optical feedback regime are strongly dependent on the feedback length from an external mirror and also on the feedback reflectivity. Variations in feedback length and strength give rise to drastic changes in the spatio-temporal extent of filaments. As result, a dramatic reduction in the excitation of higher spatial modes is found for certain ranges of feedback strength, and the number of excited spatial modes in the averaged near-field pattern is reduced. In this study, we will also investigate the spatio-temporal size of filaments and the time-averaged near-field pattern for filtered optical feedback. 


\section{THEORY}

Here, we present a theoretical model for optical feedback in a broad-area semiconductor laser. Since such a laser has a broad light emitting area along the stripe width, the effects of optical diffraction play important roles in the dynamics. Considering the spatial dependence along the stripe width, the complex laser field $E(x, t)$ ( $x$ is the coordinate along the stripe width) is given by [1]

$$
\begin{aligned}
\frac{\partial E(x, t)}{\partial t}= & i D_{e} \frac{\partial^{2} E(x, t)}{\partial x^{2}}+\frac{1}{2}(1-i \alpha) G_{n}\left\{n(x, t)-n_{t h}\right\} \\
& \times E(x, t)+\frac{\kappa}{\tau_{\text {in }}} E(x, t-\tau) \exp \left(i \omega_{0} \tau\right)+R_{s p}
\end{aligned}
$$

where $D_{\mathrm{e}}$ is the diffraction coefficient given by $D_{e}=c / 2 k_{0} \eta^{2}$ ( $c, k_{0}$, and $\eta$ being the speed of light in vacuum, the wavenumber, and the refractive index in the medium), $\alpha$ is the linewidth enhancement factor, $G_{n}$ is the gain, $n_{t h}$ is the carrier threshold, and $R_{s p}$ is a noise term induced by spontaneous emission [21]. The third term in (1) is the effect of optical feedback, in which $\kappa$ is the feedback parameter and $\tau_{\text {in }}=2 \eta l / c$ ( $l$ is the internal cavity length) is the round trip time of light inside the laser cavity. The feedback parameter is defined by [13]

$$
\kappa=\left(1-r_{2}^{2}\right) \frac{r_{3}}{r_{2}}
$$

where $r_{2}$ is the reflection coefficient of the front facet of the laser cavity and $r_{3}$ is the reflection coefficient of the external mirror. $\tau=2 L / c$ ( $L$ is the distance of the external mirror from the front facet of the laser) is the round-trip time of light in the external cavity and $\omega_{0}$ is the angular frequency of the laser oscillation.

Since we assume a short cavity, $\tau$ is shorter than the time corresponding to the relaxation oscillation frequency so that the feedback time should be less than 100 ps for ordinary broadarea semiconductor lasers. The spontaneous emission term is included in the rate equation, since actual laser oscillations contain noise effects. However, it has little effect on the essential laser dynamics so long as the noise induced by spontaneous emission is small. We employ this term in order to avoid division-by-zero problems, which are sometimes encountered in numerical simulations. Thus, the effect of the noise term on the dynamics is negligible in the present numerical simulations.

The rate equation is almost the same as that of a narrowstripe edge-emitting laser except for the first term on the righthand side of the equation. This term accounts for the diffraction effects of light in the laser cavity. Actually, the filamentation process depends sensitively on the $\alpha$-parameter and this parameter is also affected by the dynamic filamentations. However we assume a constant $\alpha$-parameter, since good agreement was previously found between theory and experimental results using a constant value of $\alpha$ [5].

Though a broad-area semiconductor laser usually oscillates at multi-longitudinal modes, treatment of a single mode such as that given here yields excellent agreement between theory and experiments, including filamentation phenomena [5]. We assume a single reflection in the external cavity in (1). In the strict sense, for the external reflectivity of more than several percent, we must take into account the effects of multiple reflec- tions. However, the behavior of chaotic semiconductor lasers can be well represented under the assumption of a single optical feedback for the external reflectivity employed here [1]. In spite of the intense beam spots associated with filaments, Kerr nonlinearities are not usually required to describe the dynamics. Instead, we may sometimes consider a gain saturation effect for intense filaments [10]. However, the model in (1) well represents the dynamics of real broad-area semiconductor lasers.

The carrier density $n(x, t)$ is also dependent on the coordinate $x$ along the stripe width and is given by

$$
\begin{aligned}
\frac{\partial n(x, t)}{\partial t}=D_{n} \frac{\partial^{2} n(x, t)}{\partial x^{2}} & +\frac{J}{e d}-\frac{n(x, t)}{\tau_{s}} \\
& -G_{n}\left\{n(x, t)-n_{0}\right\}|E(x, t)|^{2} .
\end{aligned}
$$

The first term of the right-hand side of the equation represents the carrier diffusion effect and the diffusion coefficient $D_{n}$ is given by $D_{n}=l_{d}^{2} / \tau_{s}$ ( $l_{d}$ is the diffusion length). Here, $J$ is the injection current, $e$ is the elemental charge, $d$ is the thickness of the active layer, $\tau_{s}$ is the carrier relaxation time, and $n_{0}$ is the carrier density at transparency. Due to the presence of the diffraction coefficient in the field equation and the diffusion coefficient in the carrier density equation, the laser shows a rich variety of dynamics at a microscopic scale.

We employ a simple difference method to numerically solve the rate equations. Since the equations deal with dimensions corresponding to the optical wavelength, the calculations must be carried out for time steps of much less than a femtosecond. The spatial and temporal steps for the numerical calculations are chosen to be much smaller than the optical wavelength and the optical oscillation frequency, which satisfies the von Neumann stability conditions [22]. In the simulations, we assume a stripe width for the active layer of $w=50.0 \mu \mathrm{m}(-25 \mu \mathrm{m} \leq x \leq$ $+25 \mu \mathrm{m})$. The other parameters for the numerical calculations are as follows; $l=500 \times 10^{-6} \mathrm{~m}, l_{d}=3.00 \times 10^{-6} \mathrm{~m}$, $\eta=3.59, d=0.100 \times 10^{-6} \mathrm{~m}, D_{e}=1.44 \mathrm{~m}^{2} \mathrm{~s}^{-1}, D_{n}=$ $3.00 \times 10^{-3} \mathrm{~m}^{2} \mathrm{~s}^{-1}, \alpha=3.00, G_{n}=2.00 \times 10^{-13} \mathrm{~m}^{3} \mathrm{~s}^{-1}$, $n_{\text {th }}=5.11 \times 10^{24} \mathrm{~m}^{-3}, n_{0}=1.30 \times 10^{24} \mathrm{~m}^{-3}, \tau_{\text {in }}=6.00 \times$ $10^{-12} \mathrm{~s}, \tau_{s}=3.00 \times 10^{-9} \mathrm{~s}$, optical wavelength of $\lambda=0.780 \times$ $10^{-6} \mathrm{~m}, r_{2}=0.22$, back facet reflectivity of the laser cavity $r_{1}=0.95$, and the bias injection current $J=1.5 J_{t h}\left(J_{t h}\right.$ is the threshold current). The feedback coefficient $\kappa$ and the external cavity length $L$ are variable parameters.

\section{NUMERICAL RESULTS}

\section{A. Filaments in Solitary Lasers}

Before showing the results of the dynamics for short optical feedback in broad-area semiconductor lasers, we analyze the properties of the solitary laser used in the numerical simulations. Fig. 1 shows an example of the spatio-temporal dynamics for a bias injection current of $1.5 J_{t h}$. Fig. 1(a) is an example of the spatio-temporal patterns for dynamic filamentations. The horizontal axis is the $x$ coordinate at the exit facet of the laser. Bright spots propagate irregularly in a zigzag manner as time progresses. From a detailed correlation analysis of the spatiotemporal patterns in Fig. 1(b) (see also Fig. 6), the spatial size, which is defined as the full-width at half-maximum of the correlation, is calculated to be $5.32 \mu \mathrm{m}$ and the temporal size $16.0 \mathrm{ps}$. 


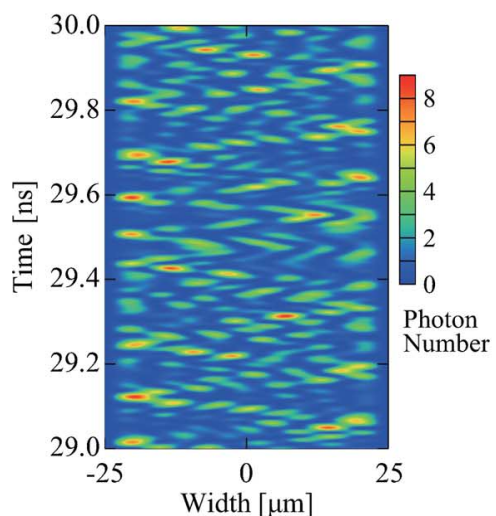

(a)

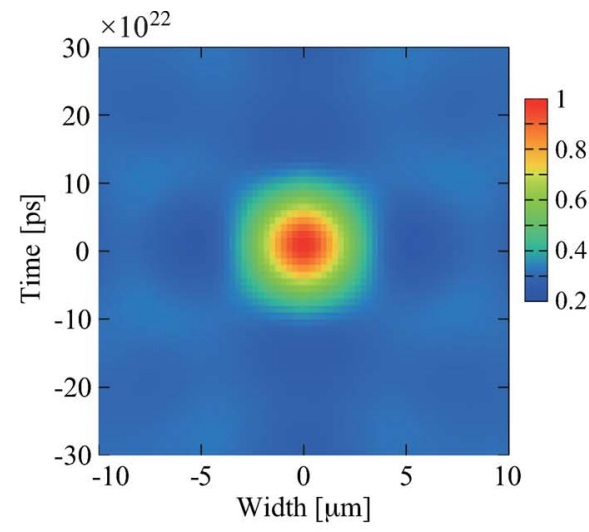

(b)

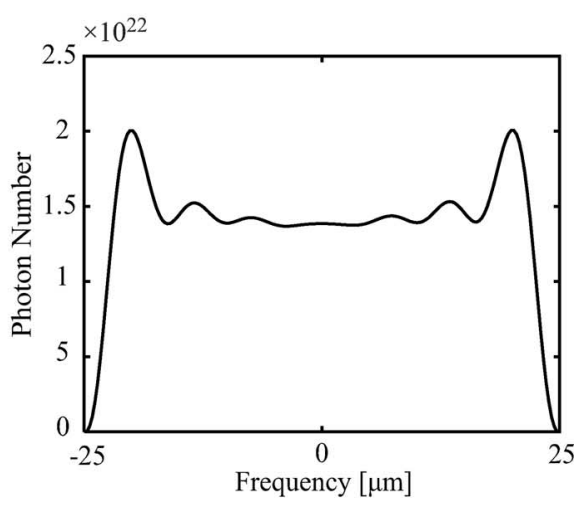

(c)

Fig. 1. (a) Spatio-temporal near-field pattern of filamentations in solitary broad-area semiconductor laser. (b) Spatio-temporal correlation of filaments. (c) Time averaged near-field pattern.

The behavior of filaments and the order of filament sizes agree well with the results of the previous experiments [5].

It is noted that bright spots frequently appear at both edges of the stripe. This agrees well with experimental results for an index-guided laser rather than a gain-guided laser [23]. Since the carrier distribution and the refractive index profile across the stripe width dynamically vary with bias injection current, it may not be easy to formulate the laser rate equation for a particular guiding model. However, the intensity profile of the twin-peaked pattern in the present simulation, which is usually observed for an index-guiding laser structure, can be explained as follows. The refractive index of a broad-area semiconductor laser is initially designed to be constant. However, during carrier injection, its value in the center of the active region decreases relative to the edge regions due to plasma effects. As a result, photons tend to be confined along both edges of the stripe. The antiguiding parameter $\alpha$ and the carrier diffusion coefficient $D_{n}$ also contribute to these dynamics.

Fig. 1(c), which is the time-averaged near-field pattern at the exit facet of the laser, clearly shows this effect. We can also see small peaks in the time-averaged intensity distribution, which correspond to excited spatial modes. The excitation of a number of higher spatial modes in broad-area semiconductor lasers by varying the bias injection current has been explained by the superposition of Hermite-Gaussian polynomials based on a steady-state model [23]. Though we did not perform a modal decomposition, closer inspection of the beam distribution makes it possible to count the number of spatial modes. From Fig. 1(c), the number of modes is determined to be 7 , which is quite consistent with previous theoretical and experimental results [23].

To investigate the irregular features of filament oscillations at the solitary mode, the intensity at the center of the stripe (i.e., $x=0 \mu \mathrm{m}$ ) is extracted and displayed in Fig. 2(a). Fig. 2(b) shows the corresponding RF spectrum. Although the spectrum is somewhat noisy, the relaxation oscillation frequency of the solitary laser can be determined to be $2.2 \mathrm{GHz}$. The peak observed at $62 \mathrm{GHz}$ is considered to correspond to dynamic filaments. The relaxation oscillation frequency of $2.2 \mathrm{GHz}$ determined from Fig. 2(b) is consistent with the value estimated from the numerical model. In addition, the frequency of $62 \mathrm{GHz}$ is
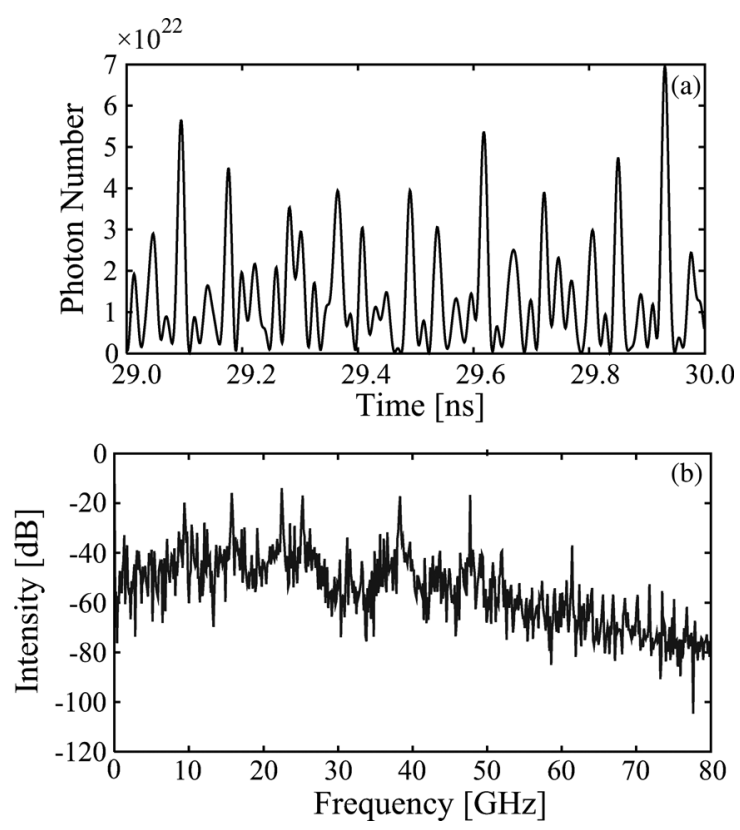

Fig. 2. (a) Time series and (b) corresponding RF spectrum at $x=0 \mu \mathrm{m}$ (center of active region) in Fig. 1, where $x$ is the coordinate along the stripe width.

consistent with the value calculated from the spatio-temporal correlation shown in Fig. 1(b). The other spectral peaks which appear in the spectrum may be the result of beats between the two components. However, since the factors determining the filament frequency and other characteristic frequencies are not well understood, a more detailed analysis of the spectral components is required.

\section{B. Results for Short Optical Feedback}

Next we consider the dynamics in broad-area semiconductor lasers with short optical feedback. We assume that the emitted light from a broad-area laser is collimated and is reflected by an external mirror, which is located at less than several centimeters away from the front facet of the laser. Thus, the emitted beam is returned to exactly the same position in the active region with a one-to-one correspondence. We show typical examples of the 

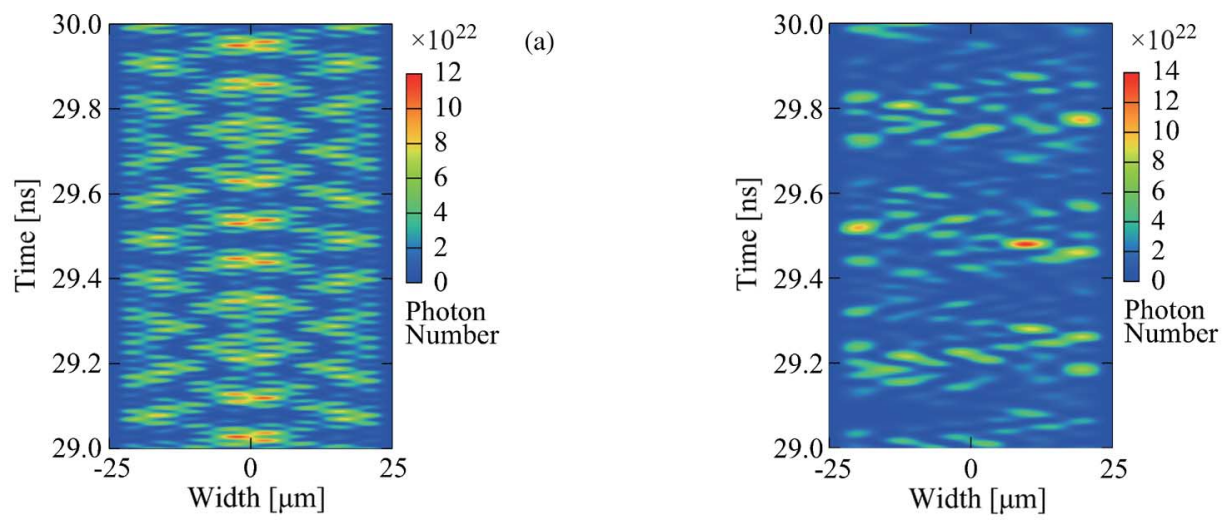

(d)
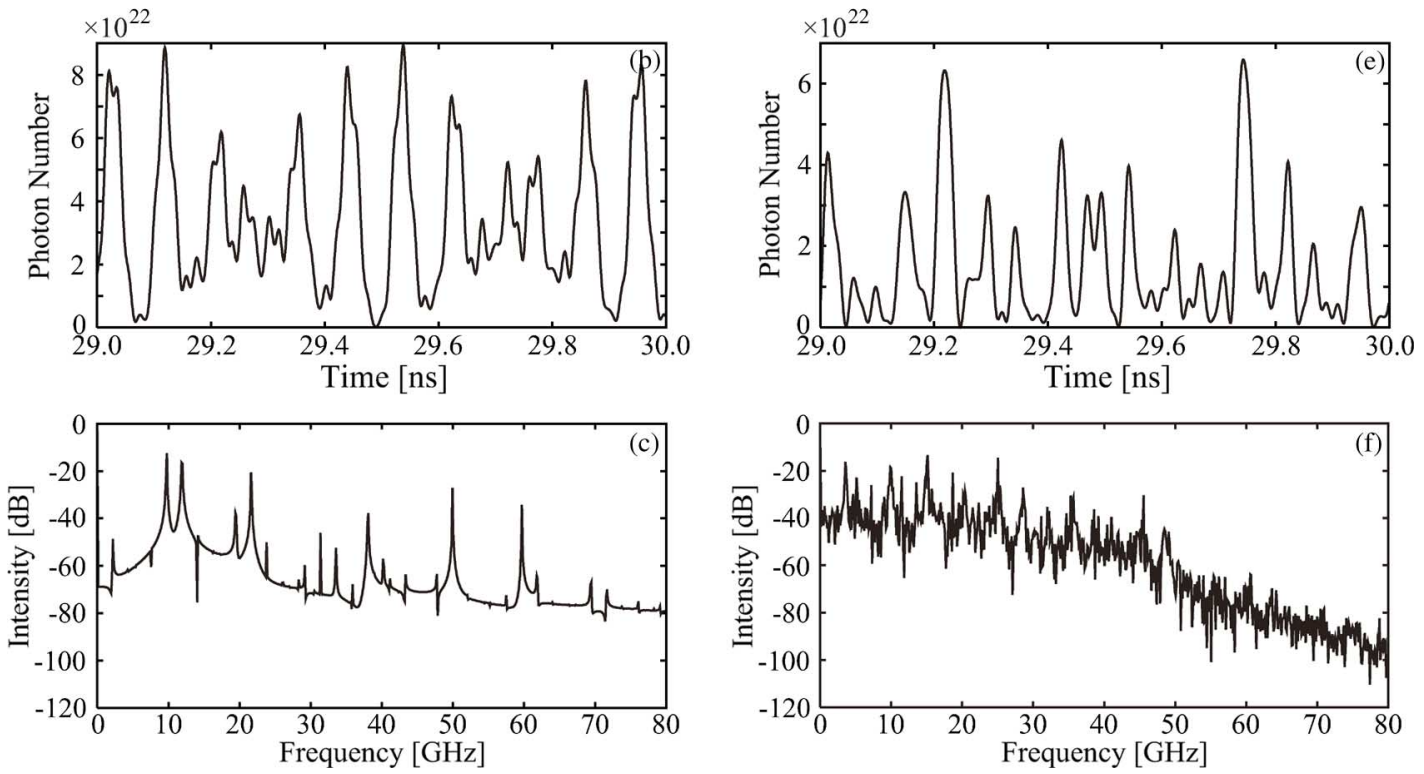

Fig. 3. Near-field pattern, time series, and corresponding RF spectrum at $x=0 \mu \mathrm{m}$ and $r_{3}=0.0125$, for $L=1.5 \mathrm{~cm}$ (left) and $L=3.0 \mathrm{~cm}$ (right).

results for external mirror distances of 1.5 and $3.0 \mathrm{~cm}$. Since the relaxation oscillation frequency of the solitary laser is about $2 \mathrm{GHz}$, which is equivalent to a mirror position of $15 \mathrm{~cm}$, the selected mirror distances are small enough to be considered as short optical feedback. Although in the numerical simulations, we can arbitrarily choose the mirror position, realistic configurations must be selected for the experimental setup. For a short external cavity length of $1.5 \mathrm{~cm}$, although it is difficult to use ordinary free space optical elements, miniature optics and/or fiber optical components can be employed.

Fig. 3 shows the results for filamentations at a feedback reflectivity of $r_{3}=0.0125$. Fig. 3(a) represents the spatio-temporal near-field pattern of filamentations. Compared with the solitary filaments in Fig. 1(a), the pattern has a periodic structure. Fig. 3(b) is a time series with a small window at the center of the near-field at $x=0 \mu \mathrm{m}$ in Fig. 3(a). In the spectrum of Fig. 3(c), which is calculated from Fig. 3(b), the lowest frequency peak can be observed at $2.2 \mathrm{GHz}$, which corresponds to a loose envelope of pulse trains and is equal to the relaxation oscillation frequency. Within this envelope, roughly periodic pulses appear with spacings of around $10 \mathrm{GHz}$, which corresponds to the round trip time of light in the external cavity. Between these pulses, fine oscillations corresponding to the frequency of the dynamic filaments can be seen. The peak around
$50 \mathrm{GHz}$ in Fig. 3(c) reveals this frequency. For the optical feedback at $3.0 \mathrm{~cm}$, the near-field pattern shows irregular filamentations and periodic structures are not distinct, although some degree of periodicity can be recognized in Fig. 3(d). Fig. 3(e) is also a time series with a small window at the center of the near-field pattern for Fig. 3(d). In the corresponding spectrum in Fig. 3(f), the lowest frequency component of $3.7 \mathrm{GHz}$ is an envelope of a rather slow periodic oscillation, which is also visible from the spatio-temporal pattern in Fig. 3(d). The spectral peak of $45 \mathrm{GHz}$ is almost equal in size to the dynamic filaments for this feedback condition. In this case, a spectral peak corresponding to the external cavity length, i.e., $5 \mathrm{GHz}$, can not be clearly distinguished.

Fig. 4 shows the results for the external reflectivity at $r_{3}=$ 0.0225 . For the short cavity of $1.5 \mathrm{~cm}$, a periodic regular pulsation state is observed as shown in Fig. 4(a) and (b). Fig. 4(b) is again a time series with a small window at the center of the near-field pattern. The package has an envelope of $10 \mathrm{GHz}$, which is equal to the time for optical feedback. Each package contains four regular pulses. The spectral peak at $40 \mathrm{GHz}$ in Fig. 4(c) corresponds to this pulse frequency. In contrast, the periodic structures for an external cavity length of $3.0 \mathrm{~cm}$ are not distinct, as shown in Fig. 4(d). However some degree of periodicity is still observable. The spectrum in Fig. 4(f), calculated 

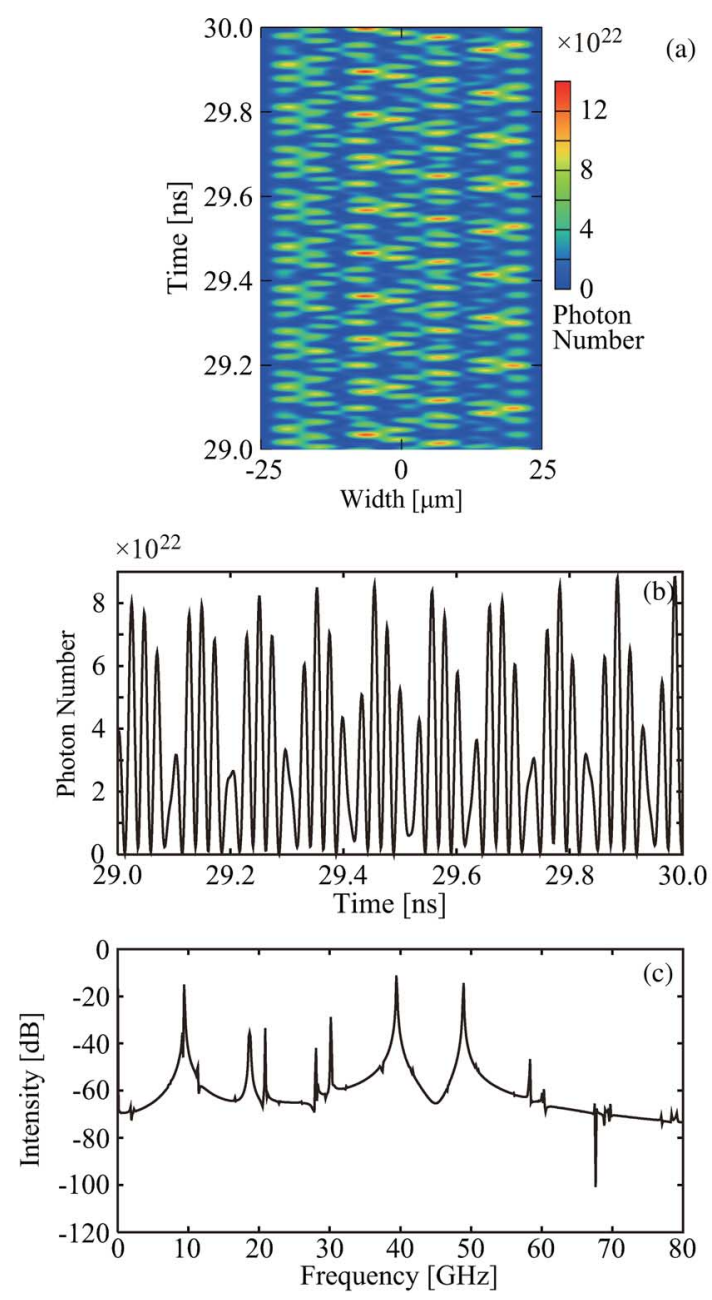

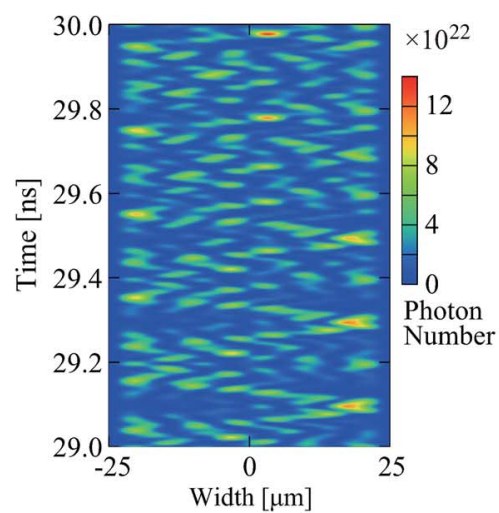

(d)
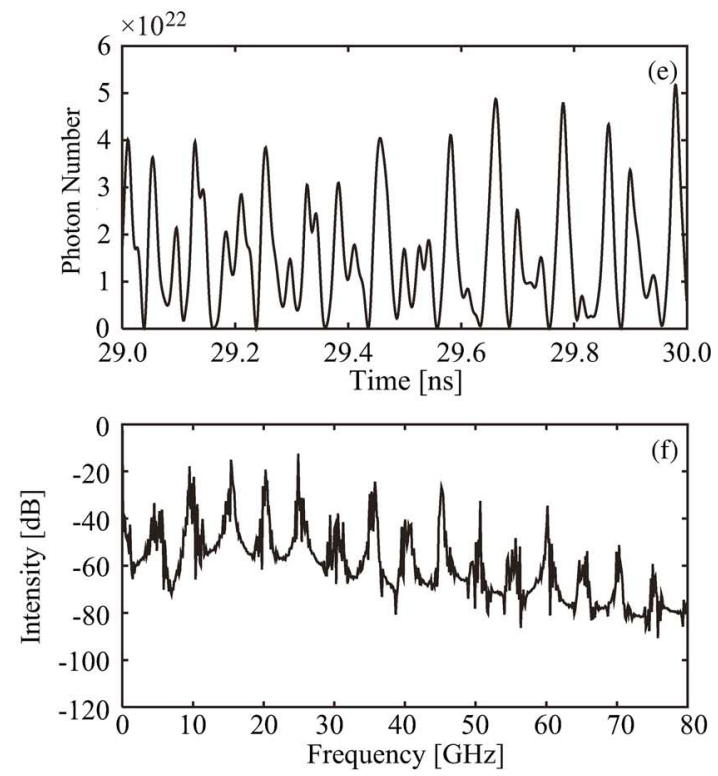

Fig. 4. Near-field pattern, time series, and corresponding RF spectrum at $x=0 \mu \mathrm{m}$ and $r_{3}=0.0225$, for $L=1.5 \mathrm{~cm}$ (left) and $L=3.0 \mathrm{~cm}$ (right).

from the time series of Fig. 4(e), includes the fundamental spectral peak at $5 \mathrm{GHz}$ and its higher harmonics. This peak is equal to the external delay of the optical feedback.

In conclusion, the periodic structure of filaments is enhanced by an increase of the feedback reflectivity, and a definite pulse package and a regular pulsation state are observed for a shorter optical feedback length. Similarly, regular pulse packages have been observed for narrow-stripe edge-emitting semiconductor lasers in short optical feedback regimes [20]. However, the pulse packages observed in broad-area semiconductor lasers are somewhat different from those for narrow-stripe edge-emitting semiconductor lasers. In the latter case, the pulse package has an envelope of a periodic frequency originated from low-frequency fluctuations of chaotic oscillations. In this case, the frequency of the pulse train inside the envelope is equal to the external feedback frequency [20]. Similar dynamics have been observed in vertical-cavity surface-emitting lasers (VCSELs) [24]. On the other hand, as discussed above, in the case of broad-area semiconductor lasers, the envelope corresponds to the external optical feedback frequency and the pulse train inside the envelope occurs at the frequency of the dynamic filaments.

Time-averaged near-field intensity profiles are next investigated as a function of external mirror reflectivity. Fig. 5 shows the results for two different external cavity lengths of $L=1.5$ and $3.0 \mathrm{~cm}$. We used a time window of $30 \mathrm{~ns}$ to obtain the averaged intensities. The near-field pattern is seen to be strongly dependent of the mirror reflectivity. As a general trend, the intensity of the peaks at both edges is reduced as the reflectivity increases. Comparing Fig. 5(a) and (b), it can be seen that the near-field pattern of the shorter external cavity strongly changes as the reflectivity increases. The number of peaks in the time-averaged intensity profile initially increases with increasing reflectivity, but the lower spatial modes are strongly excited as shown in the plot of the profile at $r_{3}=0.0125$. In this state, the spatial and temporal extent of the filaments are greatly increased, as shown in Fig. 6. For further increase of the reflectivity, the laser recovers the number of spatial modes. On the other hand, for the case of $L=3.0 \mathrm{~cm}$, the number of modes is only slightly dependent on the external reflectivity, and the intensity profile also changes in accordance with the number of modes, as shown in Fig. 5(b). However, the changes which occur in the intensity profile are rather moderate in comparison with Fig. 5(a). In the case of the shorter external cavity, the dramatic changes in the number of modes and the intensity profile may reflect an interaction between the original filament frequency and the frequency corresponding to the external optical feedback. Since these frequencies are closer together for the shorter cavity, the interference between them may be enhanced. 

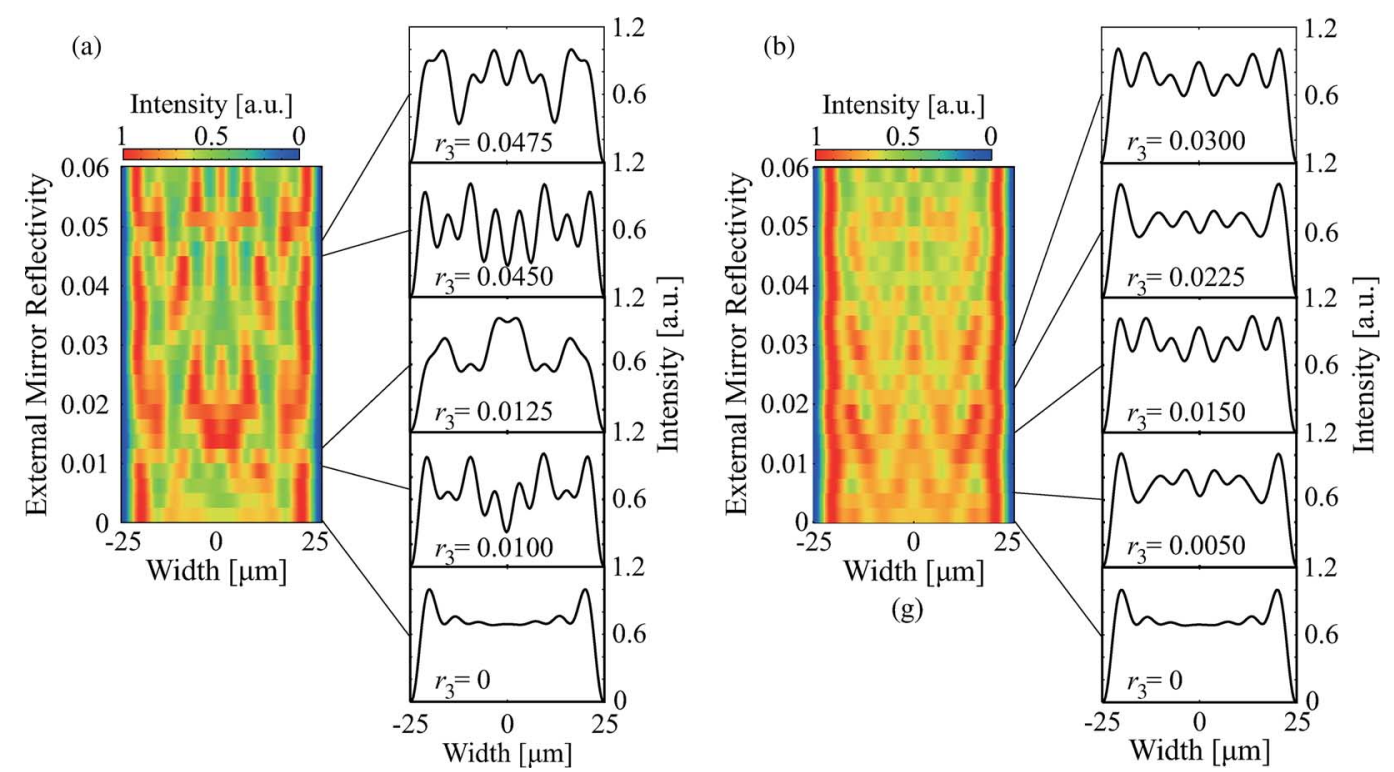

Fig. 5. Time-averaged near-field pattern for external mirror reflectivity. (a) $L=1.5 \mathrm{~cm}$, and (b) $L=3.0 \mathrm{~cm}$.
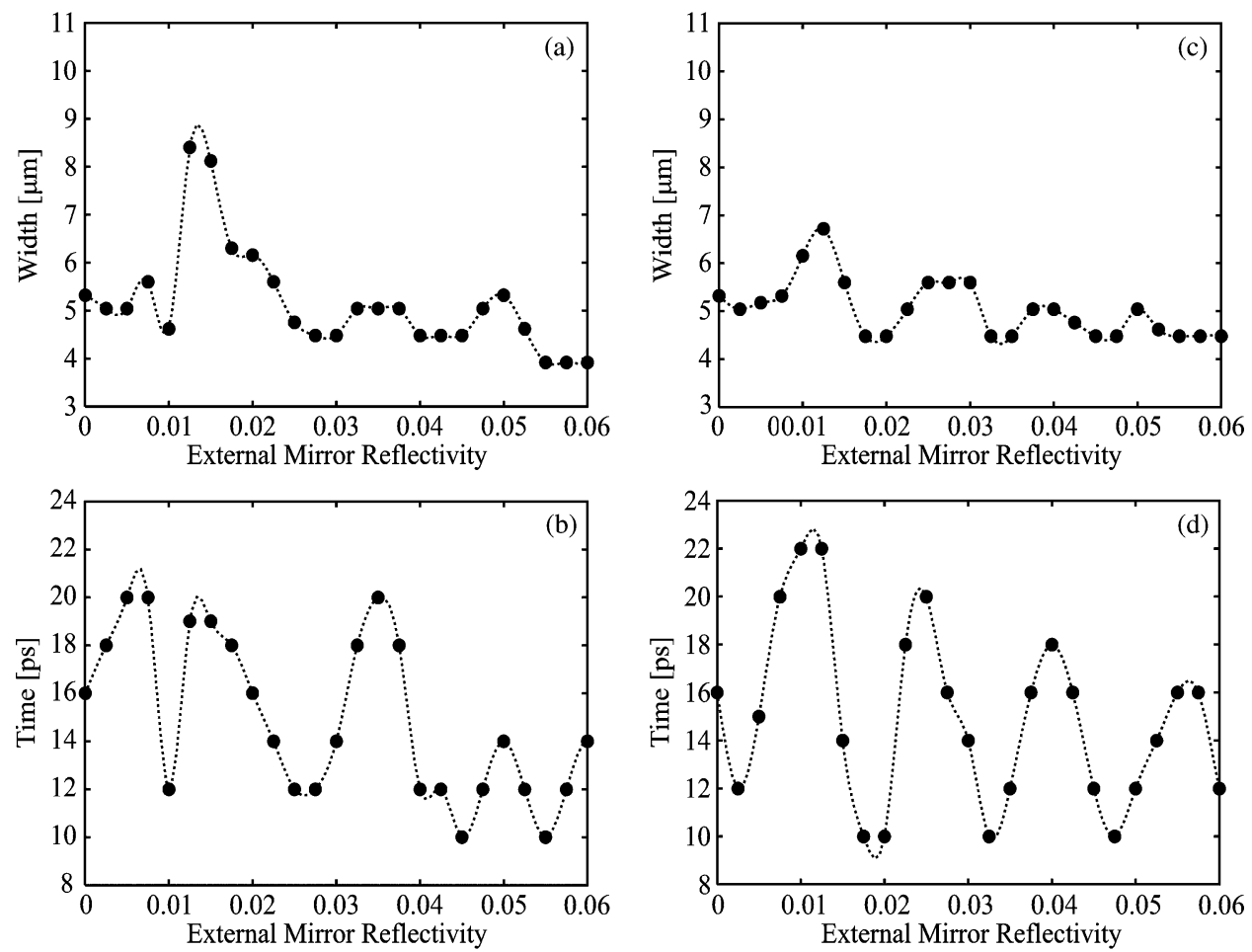

Fig. 6. Spatial and temporal filament sizes calculated from correlations. (a) Spatial size and (b) temporal size for $L=1.5 \mathrm{~cm}$, and (c) spatial size and (d) temporal size for $L=3.0 \mathrm{~cm}$.

From the spatio-temporal correlations of the near-field filament patterns, we calculated the typical spatial and temporal sizes as a function of the external mirror reflectivity. Fig. 6(a) and (b) show the case for $L=1.5 \mathrm{~cm}$, and Fig. 6(c) and (d) for $L=3.0 \mathrm{~cm}$. Both spatial and temporal filament sizes are strongly dependent on the external mirror reflectivity and a remarkable periodic structure is observed for the temporal size of the filaments. The spatial size of the filaments also has a periodic behavior which is synchronized with the period of the temporal size, though the magnitude of the variations is small. The periodic spatial variations seen in Fig. 6(a) and (c) are consistent with the results shown in Fig. 5. In the region around $r_{3}=$ 0.0125 in Fig. 6(a), where the spatial size is large, the lower spatial modes are strongly excited, as seen in Fig. 5(a), and the higher spatial modes are strongly suppressed. In the case of $L=$ $3.0 \mathrm{~cm}$, a clear periodic structure exists in the spatial size curve shown in Fig. 6(c), but the amplitude of the variation is small in accordance with the results shown in Fig. 5(b). Although the temporal sizes of the filaments exhibit the strong periodic variations shown in Fig. 6(b) and (d), this is completely averaged out 

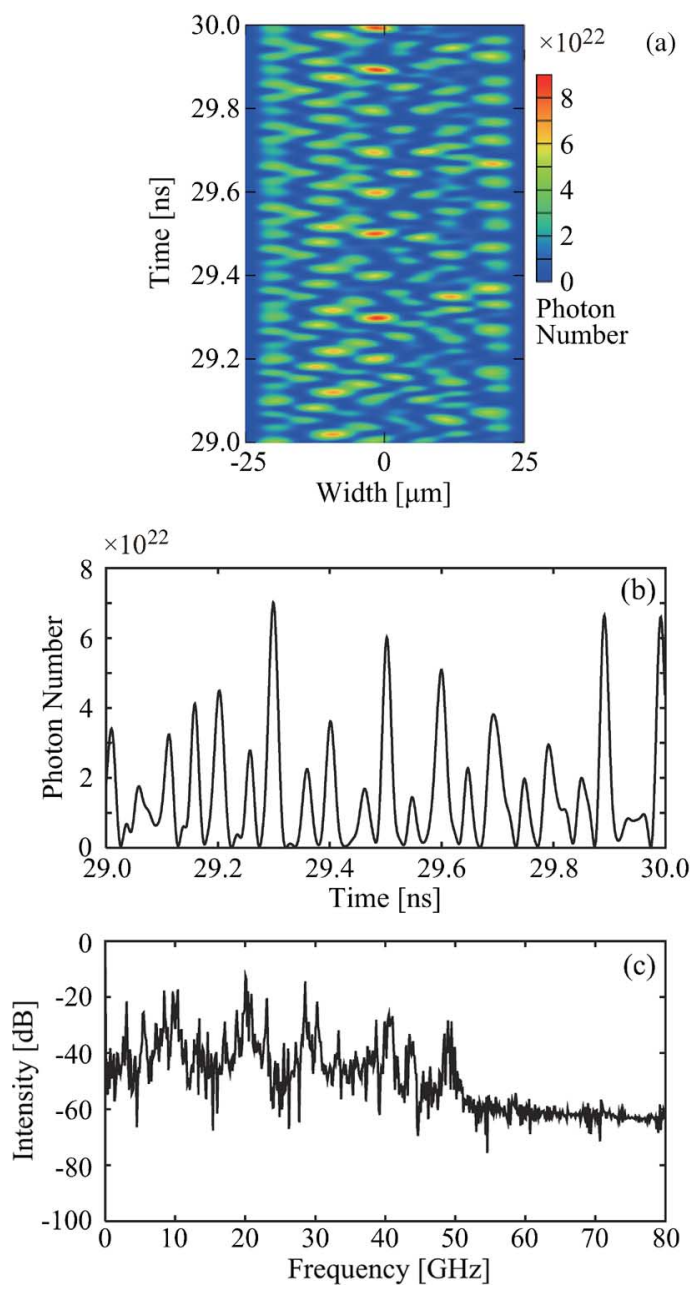

(a)

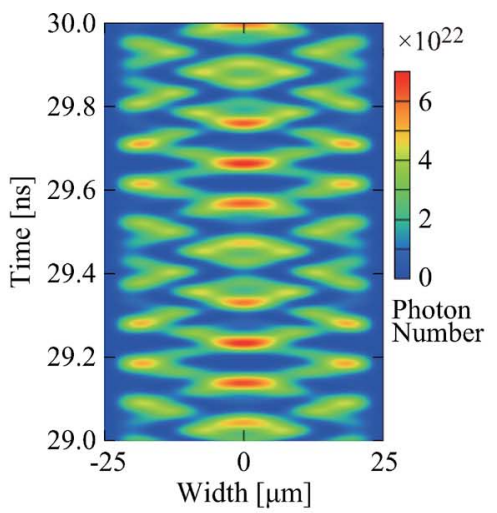

(d)
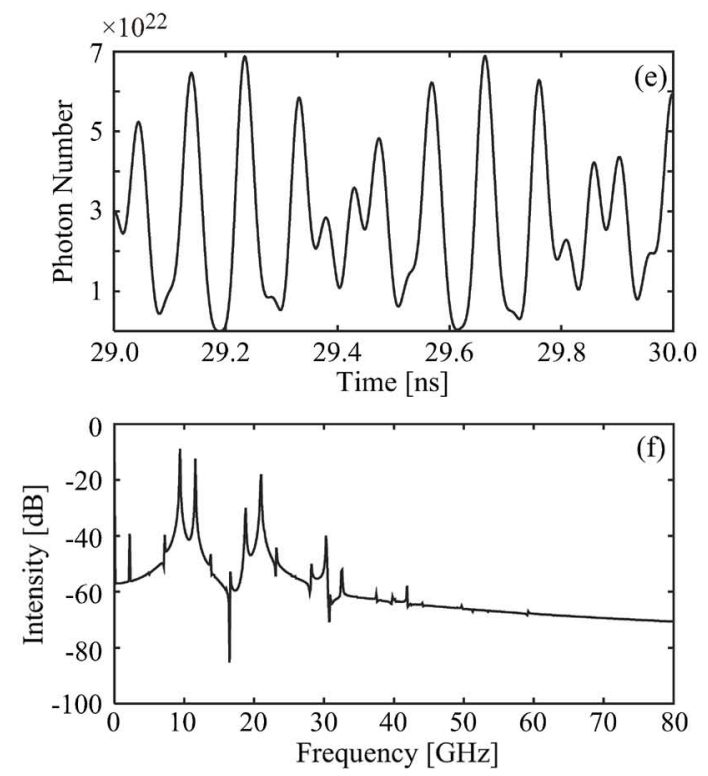

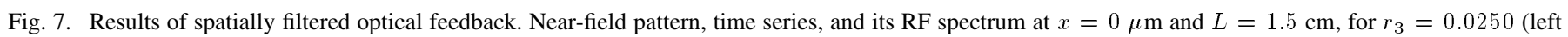
column) and $r_{3}=0.0325$ (right column).

in the static intensity profiles and little information on the temporal behavior of filaments can be obtained from the time-averaged near-field pattern. One possible reason for the strong temporal variations is the interference between the internal field and the external optical feedback in the filaments. However, further study is required to fully clarify this phenomenon.

\section{Filtered Optical Feedback}

In this subsection, we report the results for spatially filtered optical feedback. Since the number of excited higher spatial modes is dependent on the feedback intensity, optical feedback from an external reflector with a finite aperture size, i.e., optical feedback with lower order spatial modes, may control the excitation of higher spatial modes. Here we employ an external mirror whose size, $w_{e}$, at the active layer is smaller than the stripe width $\left(w_{e}<w\right)$. We tested several sizes of $w_{e}$ in combination with various optical feedback lengths. However, here we present the results of a typical case, in which we obtained characteristic features of the dynamics. The following discussion concerns with the case where $w_{e}=27.2 \mu \mathrm{m}$ and $L=1.5 \mathrm{~cm}$. The external mirror is symmetrically positioned at the center of the emitted beam.
Fig. 7 shows the results for two mirror reflectivities of $r_{3}=$ 0.0250 (left column) and $r_{3}=0.0325$ (right column). In the former case, no clear periodic structure is seen in the near-field pattern and the pattern shows irregular filamentations. The situation becomes clearer from the sampled time series at $x=0 \mu \mathrm{m}$ and its corresponding spectrum, shown in Fig. 7(b) and (c), respectively. In the spectrum, the peak at $10 \mathrm{GHz}$ corresponds to the external feedback time and peaks due to its higher harmonics are also visible. The lowest frequency peak at $2.2 \mathrm{GHz}$ corresponds to the relaxation oscillation frequency of the laser. The spectrum indicates a chaotic oscillation of the laser output. On the other hand, the periodicity is enhanced for $r_{3}=0.0325$, as shown in the right side of the figure. At the same time, the spatial and temporal sizes of the filaments have increased compared with the result for $r_{3}=0.0250$. The regular pulsations can be clearly distinguished in the time series and its corresponding spectrum, shown in Fig. 7(e) and (f), respectively. In the spectrum, the peak at $10 \mathrm{GHz}$ corresponds to the external feedback loop and the peak at $2.2 \mathrm{GHz}$ corresponds to the relaxation oscillation frequency. Higher spectral components are strongly suppressed at this reflectivity, as seen in Fig. 7(f). In fact, the periodicity is considerably enhanced within the range of optical feedback between 0.0275 and 0.0375 . Outside this 


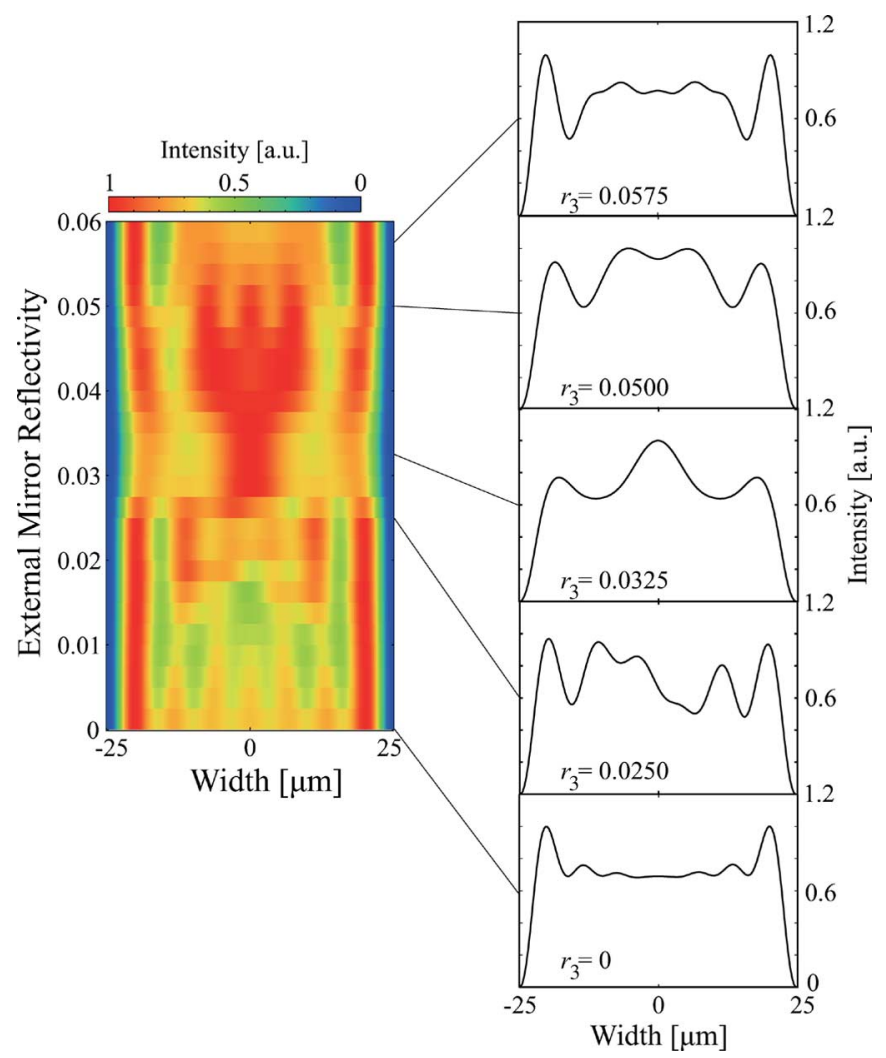

Fig. 8. Time-averaged near-field pattern of filtered optical feedback for external mirror reflectivity. The spatial filter is the same as in Fig. 7 and the external cavity length is $L=1.5 \mathrm{~cm}$.

range, the periodicity of the filaments is not clear. Additional spectra from those regions exhibit irregular frequency components of the laser oscillations.

Fig. 8 shows the time-averaged near-field pattern for the filtered optical feedback as a function of the external mirror reflectivity under the conditions of $w_{e}=27.2 \mu \mathrm{m}$ and $L=1.5 \mathrm{~cm}$. As in the case of the full width optical feedback shown in Fig. 5, the intensity peaks at the edges are initially suppressed as the external feedback reflectivity increases. The plot for $r_{3}=0.0250$ is the intensity profile just before the reduction of the number of spatial modes. At this reflectivity, the beam still contains higher spatial modes similar to that of the solitary oscillation. Within the range of reflectivity between 0.0275 and 0.0375 , the higher spatial modes are considerably reduced. The time-averaged pattern at $r_{3}=0.0325$ is a typical example of such beam profiles, in which only three intensity peaks are visible (the original pattern at $r_{3}=0$ has seven peaks). Under these conditions, the higher spatial modes are strongly suppressed and the peaks at the edges are not observable. However, with further increase of the external reflectivity (above $r_{3}=0.0375$ ), the beam recovers the higher spatial components and, at the same time, the side intensity peaks gradually grow up. These phenomena may be interpreted as a coupling and interplay of the light intensity and the carriers. Namely, for a moderate reflectivity of optical feedback, the feedback light efficiently supplies its power to carriers at the center of the active region and the laser intensity at

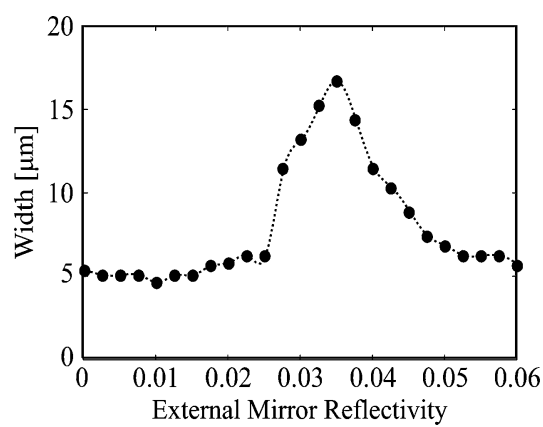

(a)

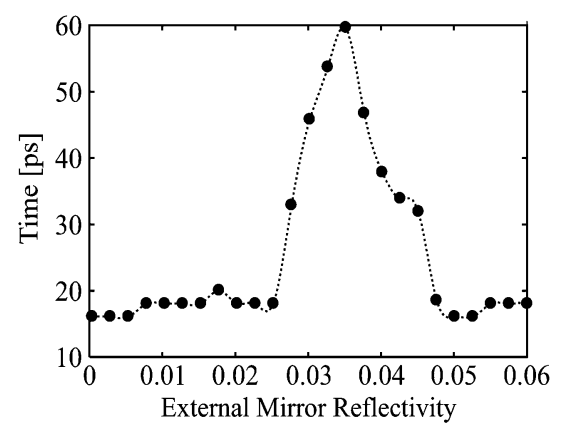

(b)

Fig. 9. (a) Spatial and (b) temporal filament sizes for external mirror reflectivity. The conditions are the same as in Fig. 7.

this area increases. As a result, the lower modes are preferentially excited. On the other hand, for strong optical feedback reflectivity, depletion of carriers through carrier hole-burning may occur and the intensity at the central area is suppressed, leading to a relative enhancement of the side peak intensities. As will be shown below, changes in the number of excited spatial modes are closely linked to the filament sizes both in space and time.

Fig. 9 shows the results for filament sizes for the filtered optical feedback. In this case, no periodic dependence on external mirror reflectivity can be observed. Instead, the filament sizes are greatly increased for a certain range of external mirror reflectivities, and are almost constant outside of this range. From Fig. 9, this range is found to be almost the same for both the spatial and temporal data. This range also appears to correspond to the region shown in Fig. 8 where little excitation of higher spatial modes occurs. Within this region, the periodicity of the laser oscillations is also enhanced, as observed in Fig. 7. Thus, from the viewpoint of beam control, filtered optical feedback may provide a suitable means for beam shaping in broad-area semiconductor lasers, although it is limited to an optimum range of optical feedback reflectivity.

\section{CONCLUSION}

We have investigated the dynamics of broad-area semiconductor lasers subjected to short optical feedback. Periodic oscillations of dynamic filaments similar to regular pulse packages observed for narrow-stripe edge-emitting semiconductor lasers have been obtained under appropriate feedback strengths. Similar periodic pulse packages have also been observed in VCSELs. However, the origin of the pulse packages in broad-area semiconductor lasers was found to be quite different from those 
in conventional edge-emitting lasers and VCSELs. Time-averaged near-field patterns have been evaluated in accordance with the filament dynamics. The excited lateral modes were found to be strongly dependent on the feedback strength and only a few lower spatial modes were excited for certain values of feedback strength. The spatio-temporal sizes of filaments showed a dependence on the feedback strength, and periodic changes of the filament sizes both in space and time was observed as a function of feedback strength.

We also investigated the dynamics for spatially filtered optical feedback regimes. By appropriate choice of filter width, the higher spatial modes were efficiently reduced and the peaks at the edges of the near-field intensity, which are typically observed for solitary oscillation, were considerably suppressed. At the same time, the spatial and temporal sizes of filaments became as much as four times larger than for the case of solitary oscillations. Thus, the sizes of dynamic filaments in broad-area semiconductor lasers can be controlled by short optical feedback. One potential application of this technique is the control and stabilization of filaments. Not only can the suppression or reduction of filament effects be expected, but also the shaping of the time-averaged laser beam. Another possibility is the application of fast pulse packages and pulsation oscillations for $\mathrm{THz}$ light sources. The temporal size of dynamic filaments is controllable by optical feedback and external optical injection. Therefore, periodic pulsating oscillations as short as picoseconds can be obtained by such techniques [19]. Since broad-area semiconductor lasers have relatively intense beams, it is possible to obtain rather large RF electromagnetic power levels, even if the excitation percentage is very small.

\section{REFERENCES}

[1] J. Ohtsubo, Semiconductor Lasers: Stability, Instability and Chaos, 2nd ed. Berlin, Germany: Springer-Verlag, 2008.

[2] E. Gehrig and O. Hess, Spatio-Temporal Dynamics and Quantum Fluctuations in Semiconductor Lasers. Heidelberg, Germany: SpringerVerlag, 2003.

[3] H. Adachihara, O. Hess, E. Abraham, P. Ru, and J. V. Moloney, "Spatiotemporal chaos in broad-area semiconductor lasers," J. Opt. Soc. Am. $B$, vol. 10, pp. 658-665, 1993.

[4] J. R. Marciante and G. P. Agraval, "Spatio-temporal characteristics of filamentation in broad-area semiconductor lasers," IEEE J. Quantum Electron., vol. 33, no. 7, pp. 1174-1179, Jul. 1997.

[5] I. Fischer, O. Hess, W. Elsäßer, and E. Göbel, "Complex spatio-temporal dynamics in the near-field of a broad-area semiconductor laser," Europhys. Lett., vol. 35, pp. 579-584, 1996.

[6] J. R. Marciante and G. P. Agraval, "Spatio-temporal characteristics of filamentation in broad-area semiconductor lasers: Experimental results," IEEE Photon. Technol. Lett., vol. 10, no. 1, pp. 54-56, 1998.

[7] T. Burkhard, M. O. Ziegler, I. Fischer, I, and W. Elsäßer, "Spatio-temporal dynamics of broad area semiconductor lasers and its characterization," Chaos, Solitons \& Fractals, vol. 10, pp. 845-850, 1999.

[8] O. Hess and T. Kuhn, "Maxwell-Bloch equations for spatially inhomogeneous semiconductor lasers. I. Theoretical formulation," Phys. Rev. A, vol. 54, pp. 3347-3359, 1996.

[9] O. Hess and T. Kuhn, "Maxwell-Bloch equations for spatially inhomogeneous semiconductor lasers. II. Spatiotemporal dynamics," Phys. Rev. A, vol. 54, pp. 3360-3368, 1996.

[10] J. R. Marciante and G, P. Agraval, "Lateral spatial effects of feedback in gain-guided and broad-area semiconductor lasers," IEEE J. Quantum Electron., vol. 32, no. 4, pp. 1630-1635, Apr. 1996.
[11] Y. Champagne, S. Mailhot, and N. McCarthy, "Numerical procedure for the lateral-mode analysis of broad-area semiconductor lasers with an external cavity," IEEE J. Quantum Electron., vol. 31, no. 5, pp. 795-810, May 1995.

[12] C. Simmendinger and O. Hess, "Controlling delay-induced chaotic behavior of a semiconductor laser with optical feedback," Phys. Lett. A, vol. 216, pp. 97-105, 1996.

[13] C. Simmendinger, D. Preißer, and O. Hess, "Stabilization of chaotic spatiotemporal filamentation in large broad area lasers by spatially structured optical feedback," Opt. Expr., vol. 5, pp. 48-54, 1999.

[14] S. K. Mandre, I. Fischer, and W. Elsäßer, "Spatiotemporal emission dynamics of a broad-area semiconductor laser in an external cavity: Stabilization and feedback-induced instabilities," Opt. Commun., vol. 244, pp. 355-365, 2005.

[15] Y. Fujita and J. Ohtsubo, "Optical-feedback-induced stability and instability in broad-area semiconductor lasers," Appl. Phys. Lett., vol. 87, pp. 031112-1-031112-3, 2005.

[16] J. Ohtsubo, "Chaos and control in semiconductor lasers," in Handbook of Chaos Control, E. Schöll and H. G. Schuster, Eds. Weinheim, Germany: Wiley-VHC, 2008, ch. 22.

[17] S. K. Mandre, I. Fischer, and W. Elsäßer, "Control of the spatiotemporal emission on a broad-area semiconductor laser by spatially filtered feedback," Opt. Lett., vol. 28, no. 13, pp. 1135-1137, 2003.

[18] N. Gaciu, E. Gehring, and O. Hess, "Control of broad-area laser dynamics with delayed optical feedback," in Handbook of Chaos Control, E. Schöll and H. G. Schuster, Eds. Weinheim, Germany: Wiley-VHC, 2008, ch. 20.

[19] S. Takimoto, T. Tachikawa, R. Shogenji, and J. Ohtsubo, "Control of spatio-temporal dynamics of broad-area semiconductor lasers by strong optical injection," IEEE Photon. Technol. Lett., vol. 21, no. 15, pp. 1051-1053, 2009.

[20] T. Heil, I. Fischer, and W. Elsäßer, "Dynamics of semiconductor lasers subject to delayed optical feedback: The short cavity regime," Phys. Rev. Lett., vol. 24, pp. 243901-1-243901-4, 2001.

[21] K. Peterman, Laser Diode Modulation and Noise. Tokyo, Japan: Kluwer Academic, 1991.

[22] M. L. Wilkins, Computer Simulation of Dynamic Phenomena. Berlin, Germany: Springer-Verlag, 1999.

[23] T. Asatsuma, Y. Takiguchi, S. Frederico, A. Furukawa, and S. Hirata, "Successive phase change and stability of near-field patterns for broadarea laser diodes," in Proc. SPIE, 20069, vol. 6104, p. 61040C.

[24] A. Tabaka, M. Peil, M. Sciamanna, I. Fischer, W. Elsäßer, H. Thienpont, I. Veretennicoff, and K. Panajotov, "Dynamics of vertical-cavity surface-emitting lasers in the short external cavity regime: Pulse packages and polarization mode competition," Phys. Rev. A, vol. 73, pp. 013810-1-013810-14, 2006.

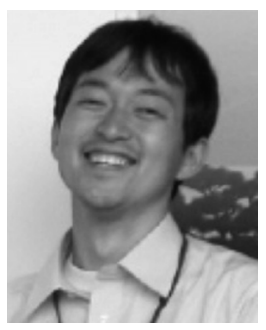

Tomokazu Tachikawa was born in Belém, Brazil. He received the B.S. and M.S. degrees in systems engineering from Shizuoka University, Shizuoka, Japan, in 2007 and 2009, respectively.

$\mathrm{He}$ is presently working at Aishin Seiki Co., Ltd. His research interests include optical engineering and laser physics, particularly in controlling instability in high-power broad-area semiconductor lasers.

Mr. Tachikawa is a member of the Japanese Society of Applied Physics and the Laser Society of Japan.

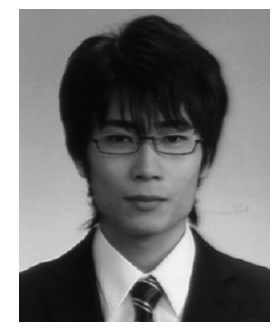

Satoshi Takimoto was born in Hamamatsu, Japan. $\mathrm{He}$ received the B.S. degree in systems engineering from Shizuoka University, Shizuoka, Japan, in 2008. $\mathrm{He}$ is currently working toward the M.S. degree in systems engineering at Shizuoka University.

His research interests include the dynamics of optical injection and instability control in high-power broad-area semiconductor lasers.

Mr. Takimoto is a member of the Japanese Society of Applied Physics and the Laser Society of Japan. 


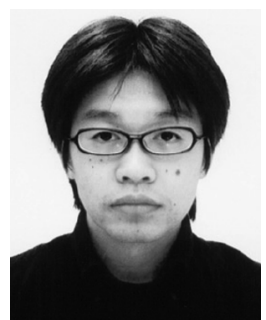

Rui Shogenji received the B.S. and M.S. degrees in computer science from the Kitami Institute of Technology, Japan, in 1999 and 2001, respectively, and the Ph.D. degree from Osaka University, Japan, in 2005.

$\mathrm{He}$ is currently an Assistant Professor in the Department of Systems Engineering, Shizuoka University, Shizuoka, Japan. His research interests include information optics, optical information processing and computing, and information hiding.

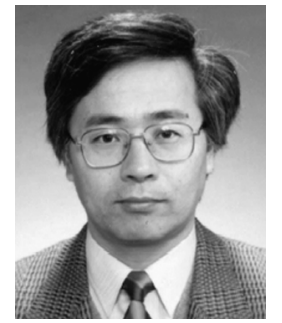

Junji Ohtsubo (M'96) received the B.S. degree in electronics from the Kyushu Institute of Technology in 1973, and the M.S. and Ph.D. degrees in electronics from Hokkaido University in 1975 and 1978, respectively.

In 1978, he joined the Mechanical Engineering Laboratory, MITI, Japan. During 1981-1982, he was a Research Associate at the Institute of Optics, University of Rochester. He joined Shizuoka University, Shizuoka, Japan, as an Associate Professor in 1985 and is presently a Professor in the Department of Systems Engineering. His current research interests are nonlinear dynamics in optics, chaos in semiconductor lasers, optical information processing and computing, optical security systems, statistical optics, speckle, and optical metrology.

Prof. Ohtsubo is a Fellow of the Optical Society of America and a member of IEEE, SPIE, AIP, the Japanese Society of Applied Physics, the Optical Society of Japan, and the Laser Society of Japan. 